\title{
Avaliação qualitativa sobre o que os psiquiatras brasileiros esperam das classificações diagnósticas
}

\author{
A qualitative analysis on what Brazilian psychiatrists expect from current diagnostic classifications
}

\author{
Amilton dos Santos Júnior ${ }^{1}$, Luiz Fernando Almeida Lima e Silva², Cláudio Eduardo Muller Banzato ${ }^{3}$, \\ Mário Eduardo Costa Pereira ${ }^{3}$
}

${ }^{1}$ Médico psiquiatra. Mestrando, Saúde da Criança e do Adolescente, Universidade Estadual de Campinas (Unicamp), Campinas, SP. ${ }^{2}$ Médico psiquiatra, Unidade de Emergência Referenciada, Hospital das Clínicas, Faculdade de Ciências Médicas, Unicamp. ${ }^{3}$ Professor livre-docente, Faculdade de Ciências Médicas, Unicamp.

Apoio financeiro e logístico: Associação Brasileira de Psiquiatria (ABP).

\section{Resumo}

Introdução: Pretende-se analisar o perfil de respostas qualitativas a um questionário padronizado anônimo, com itens abertos e fechados, sobre a percepção dos psiquiatras brasileiros acerca do uso dos sistemas diagnósticos multiaxiais CID-10 e DSM-IV e de suas expectativas quanto às próximas revisões (CID-11 e DSM-V).

Método: O questionário, elaborado por Graham Mellsop (Nova Zelândia), foi traduzido e enviado para 1.050 psiquiatras afiliados à Associação Brasileira de Psiquiatria.

Resultados: Retornaram 160 questionários (15,2\%). Nestes, 71,1\% das questões abertas foram respondidas. As principais qualidades julgadas necessárias ou desejadas em uma classificação psiquiátrica foram: simplicidade, clareza de critérios, objetividade, compreensibilidade, confiabilidade e facilidade de uso. O eixo I da CID-10 foi citado como o mais utilizado pelo caráter instrumental e pelo fato de ser a classificação oficial, inclusive para fins burocráticos e legais. O DSM-IV também é bastante utilizado no cotidiano, sobretudo para ensino e pesquisa, por psiquiatras com afiliações acadêmicas. $\mathrm{O}$ uso menos frequente de sistemas multiaxiais foi justificado pela falta de treino e de familiaridade, pela sobrecarga de informações e pelo fato de não serem obrigatórios. Avaliou-se que algumas categorias diagnósticas, entre outras, devem ser revistas, como: retardo mental, transtornos alimentares, de personalidade, do sono, da infância e adolescência, quadros ansiosos, afetivos, esquizoafetivos.

Conclusão: O material fornece um panorama sobre as opiniões e expectativas dos psiquiatras a respeito dos instrumentos diagnósticos dos quais se servem ativamente em sua prática diária e cujas revisões estão em pleno processo de elaboração.

Descritores: Classificação, diagnóstico, utilidade clínica, sistemas multiaxiais.

\begin{abstract}
Introduction: The objective of the present study was to analyze the qualitative answers of an anonymous standardized survey including qualitative and quantitative questions about the Brazilian psychiatrists' perceptions on their use of the multiaxial diagnostic systems ICD-10 and DSM-IV and about their expectations regarding future revisions of these classifications (ICD-11 and DSM-V).

Method: The questionnaire, elaborated by Graham Mellsop (New Zealand), was translated into Portuguese and sent through mail to 1,050 psychiatrists affiliated to the Brazilian Psychiatry Association. The quantitative analysis is presented elsewhere.

Results: One hundred and sixty questionnaires returned (15.2\%). From these, $71.1 \%$ of the open questions where answered. The most needed and/or desirable qualities in a psychiatric classification were found to be: simplicity, clarity of criteria, objectivity, comprehensibility, reliability, and ease to use. Axis I of the ICD-10 was reported to be the most used due to its instrumental character in addition to being the official classification also for legal and bureaucratic purposes. The DSM-IV was also used in the everyday practice, mostly for education and research purposes, by psychiatrists with academic affiliations. The less frequent use of the multiaxial systems was explained by the lack of training and familiarity, the overload of information and by the fact they are not mandatory. Based on the respondents' answers, we concluded that some diagnostic categories must be revised, such as: mental retardation, eating disorders, personality disorders, sleeping disorders, child and adolescent disorders, affective, and schizoaffective disorders.

Conclusion: This material offers a systematic overview of the psychiatrists' opinions and expectations concerning the diagnostic instruments used in their daily practice.
\end{abstract}

Keywords: Classification, diagnosis, clinical utility, multiaxial systems.

Correspondência:

Amilton dos Santos Júnior, Rua Joaquim Fogaça, 293, CEP 18114-240, Bairro Dominguinho, Votorantim, SP. Tel.: (19) 3521.7206 , (15) 3241.1177, (19) 9721.1585. E-mail: amilton1983@yahoo.com.br

Não há conflitos de interesse associados à publicação deste artigo.

Copyright (C) Revista de Psiquiatria do Rio Grande do Sul - APRS

Recebido em 19/12/2008. Aceito em 24/02/2009. 


\section{Introdução}

No momento em que os primeiros passos estão sendo dados para a elaboração da Classificação Estatística Internacional de Doenças e Problemas Relacionados à Saúde (CID), $11^{\mathrm{a}}$ revisão, e do Manual Diagnóstico e Estatístico de Transtornos Mentais (DSM), quinta revisão, a discussão sobre o futuro das classificações diagnósticas em psiquiatria tem se desenvolvido em torno de dois parâmetros básicos: validade e utilidade ${ }^{1-3}$. Embora a ambição científica que motiva as classificações diagnósticas - especialmente o sistema DSM - venha recebendo mais atenção da literatura especializada, sempre houve uma preocupação a respeito do impacto clínico e educacional da adoção e do uso de tais sistemas ${ }^{1,4-7}$. Desde o lançamento do DSM-III (1980), um marco na psiquiatria por ser a primeira classificação baseada em critérios diagnósticos explícitos e a primeira que adotou oficialmente um sistema multiaxial ${ }^{8}$, várias pesquisas têm sido realizadas, especialmente nos EUA e no Canadá, sobre o uso e o impacto clínico e sobre o ensino do DSM-III e de seus sucessores, com diferentes populações e em diferentes contextos ${ }^{1,4-6,9,10}$.

Os objetivos do presente estudo são investigar o uso e as percepções dos psiquiatras brasileiros sobre a utilidade das principais classificações diagnósticas atuais (CID-10 e DSM-IV) e identificar suas expectativas sobre os futuros sistemas de classificação. Mais especificamente, pretende-se verificar os atributos desejáveis para uma classificação diagnóstica de acordo com a opinião dos participantes da pesquisa. Levantamentos anteriores focaram somente o uso e a utilidade percebida das classificações diagnósticas. Neste estudo, também se consideraram as expectativas e algumas preferências taxonômicas dos psiquiatras com relação aos sistemas de classificação.

\section{Método}

O questionário utilizado é a tradução para o português do instrumento original em inglês elaborado por Graham Mellsop (Nova Zelândia). Realizou-se uma retrotradução independente para o inglês e uma comparação para avaliar sua equivalência semântica com o questionário original. Também foram realizadas revisões da tradução quando necessário.

Efetuou-se uma análise detalhada das respostas dos psiquiatras brasileiros a esse questionário ${ }^{1}$. Há outro estudo em que esses dados foram combinados com aqueles coletados por Graham Mellsop na Nova Zelândia e com os resultados de um terceiro levantamento, realizado no Japão, utilizando o mesmo questionário ${ }^{11}$. Esse estudo foi realizado para identificar as características globais dos sistemas diagnósticos e os sistemas de classificação preferidos pelos profissionais de três países com diferenças culturais, socioeconômicas e geográficas $^{11}$.

No presente relato, somente as respostas dos psiquiatras brasileiros para as perguntas abertas do questionário foram consideradas, as quais são articuladas com elementos quantitativos do instrumento, e solicitou-se que os participantes listassem as qualidades e os atributos considerados necessários ou desejáveis para uma classificação psiquiátrica, além de comentarem as razões pelas quais rotineiramente utilizam ou nunca utilizam certos eixos do DSM-IV e da CID-10 e apontarem quaisquer diagnósticos específicos do DSM-IV ou da CID-10 que deveriam ser retirados ou deslocados para outra seção.

A partir da lista de correspondência do Departamento de Diagnóstico e Classificação em Psiquiatria da Associação Brasileira de Psiquiatria (DDCP-ABP), contendo os nomes de 3.062 psiquiatras afiliados, 1.050 foram selecionados aleatoriamente para receber o questionário e um envelope selado para a devolução. Após o envio dos questionários, duas mensagens eletrônicas foram enviadas (na segunda e na quinta semanas posteriores) aos endereços eletrônicos dos psiquiatras incluídos na lista original para reforçar a solicitação de preenchimento da pesquisa. Não houve identificação do psiquiatra no questionário.

\section{Resultados}

Da amostra total de 1.050 questionários, 21 não puderam ser entregues aos destinatários devido a mudança de endereço ou endereço incompleto ${ }^{1}$. Cento e sessenta questionários foram devolvidos, o que representa uma taxa de resposta de $15,2 \%{ }^{1,11}$. Entre os questionários devolvidos, $71,1 \%$ das questões abertas foram respondidas.

Quando solicitados a listar as qualidades ou atributos necessários ou desejados para uma classificação psiquiátrica, a maioria dos psiquiatras $(75 \%$ - 120 dos 160 questionários devolvidos) concordou sobre a importância de critérios claros, simples, objetivos, compreensíveis, confiáveis e fáceis de utilizar. Esses foram considerados itens importantes para uma melhor explicação dos sintomas e das estruturas psicopatológicas, para informar sobre o tratamento e para indicar possíveis etiologias e patogenias. Alguns exemplos das respostas mais frequentes incluíram:

Clareza, objetividade, orientação de escolhas, prognóstico e terapêutica.

Boa validade e confiabilidade, fácil aplicação, compreensão e critérios claros.

Simplificação, com ênfase nos sintomas principais/nucleares da patologia.

Após uma investigação sobre o uso de cada eixo do DSM-IV e da CID-10 (Tabela 1), solicitou-se que os psiquiatras comentassem a razão do uso rotineiro desses instrumentos. Para cinco dos 160 participantes $(3,1 \%)$, a questão não se aplicou porque eles informaram que não faziam uso 
Tabela 1 - Uso dos sistemas diagnósticos

\begin{tabular}{lccc}
\hline & Uso rotineiro (\%) & Uso eventual (\%) & Ausência de uso (\%) \\
\hline Eixo I do DSM-IV $(\mathrm{n}=136 ; 85,0 \%)$ & 55,9 & 23,5 & 20,6 \\
Eixo II do DSM-IV $(\mathrm{n}=135 ; 84,4 \%)$ & 39,3 & 37,0 & 23,7 \\
Eixo III do DSM-IV $(\mathrm{n}=135 ; 84,4 \%)$ & 31,1 & 34,8 & 34,1 \\
Eixo IV do DSM-IV $(\mathrm{n}=133 ; 83,1 \%)$ & 15,8 & 39,8 & 44,4 \\
Eixo V do DSM-IV $(\mathrm{n}=130 ; 81,3 \%)$ & 11,5 & 40,0 & 48,5 \\
CID-10 - eixo de diagnóstico clínico $(\mathrm{n}=154 ; 96,3 \%)$ & 83,1 & 13,0 & 3,9 \\
CID-10 - eixo de funcionalidade/incapacitações $(\mathrm{n}=132 ; 82,5 \%)$ & 30,3 & 42,2 & 26,5 \\
\hline
\end{tabular}

rotineiro de nenhum dos eixos listados na Tabela 1. Entre o restante, a solicitação foi discutida por 134 psiquiatras (86,5\%). O eixo I da CID-10 foi mencionado como o mais utilizado. A razão mais frequente foi relacionada ao fato de que essa é a classificação oficial, além de um "instrumento de comunicação com outros profissionais, leigos, a justiça e as administradoras de planos de saúde", o que "possibilita a comunicação com toda a equipe do serviço de saúde".

O DSM-IV também é amplamente utilizado (principalmente o eixo I), geralmente pelo mesmo profissional que declarou utilizar a CID-10. Aqueles que fazem uso rotineiro do DSM-IV mencionaram as seguintes razões: afiliações acadêmicas, para uso junto a estudantes e residentes (pois é a classificação encontrada em muitas fontes da literatura), ou por ser um sistema que permite formulações diagnósticas amplas.

Com relação às razões para não utilizar alguns eixos dos sistemas de classificação, 30 participantes mencionaram o uso, ainda que ocasional, de todos os eixos da CID-10 e do DSM-IV. Portanto, essa pergunta não se aplicou a eles. Entre os outros 130 psiquiatras que responderam esse item, $97(74,6 \%)$ comentaram a solicitação, referindo a falta de treino e familiaridade, o desconhecimento sobre os critérios de classificação e a pouca praticidade como sendo as razões mais importantes para não utilizarem os eixos mencionados. Tais comentários foram mais frequentemente apresentados como uma explicação para a ausência do uso das classificações multiaxiais.

A última questão aberta investigou se havia categorias diagnósticas que os psiquiatras consideravam que devessem ser retiradas dos instrumentos ou deslocadas para outra seção do DSM-IV e da CID-10. De acordo com os 104 participantes que responderam essa pergunta $(65 \%$ dos questionários devolvidos), 33 (31,7\%) responderam "não" e $12(11,5 \%)$ responderam que não sabiam opinar a respeito, não se sentiam capazes de comentar ou nunca haviam pensado sobre a questão. Os outros 59 psiquiatras $(56,7 \%)$ consideraram que algumas categorias diagnósticas são problemáticas e devem ser revistas. As categorias mais citadas foram:

- transtornos neuróticos, relacionados a estresse e somatoformes (muitas categorias, com estruturas que se sobrepõem e com considerável associação com transtornos de humor);

- transtornos de personalidade (dificuldades para diagnosticar e delimitar os subtipos);

- transtornos de humor persistentes (distimia/ciclotimia);

- transtorno obsessivo compulsivo (com várias sugestões para que seja separado de outros transtornos de ansiedade);

- transtorno esquizoafetivo (questionamentos sobre a validade do construto);

- transtorno esquizotípico (falta de consenso: diagnóstico de transtorno do eixo clínico na CID versus transtorno de personalidade no eixo II do DSM-IV);

- transtorno de humor bipolar (separação de depressão unipolar, debates sobre o construto do "espectro bipolar");

- outros: transtornos com início na infância; transtorno do controle do impulso, retardo mental, esquizofrenia simples, transtornos alimentares e transtornos do sono.

\section{Discussão}

Simplicidade, clareza de critérios, objetividade, confiabilidade, compreensibilidade e facilidade de uso estão entre os atributos-chave considerados necessários para uma classificação psiquiátrica.

O sistema diagnóstico mais utilizado pelos psiquiatras brasileiros é a CID-10 (pelo menos seu eixo I) devido ao fato de ser uma classificação internacional e a adotada oficialmente no país para fins burocráticos e legais. O eixo I do DSM-IV é utilizado rotineiramente em ambientes ligados a ensino e pesquisa. $\mathrm{O}$ uso menos frequente dos outros eixos 
dos sistemas multiaxiais atuais foi justificado pela falta de treino e familiaridade, pela falta de conhecimento sobre os critérios de classificação e pela menor praticidade.

Grande parte dos psiquiatras que responderam a pesquisa considerou que as categorias diagnósticas deveriam ser revistas ou deslocadas para outra seção, com base nos sistemas de classificações atuais.

Embora não seja possível afirmar que essas respostas representam a opinião de todos os psiquiatras brasileiros, devido à taxa relativamente baixa de questionários devolvidos, esses dados oferecem um panorama amplo e fértil das opiniões e expectativas dos psiquiatras brasileiros sobre as ferramentas diagnósticas disponíveis em sua prática diária e cujas revisões estão em pleno processo de elaboração ${ }^{1,11}$.

\section{Conclusão}

Aparentemente, os médicos psiquiatras preferem critérios diagnósticos simples, úteis e de fácil utilização em sua prática diária, o que de certa forma contraria a tendência divisória e de expansão de categorias classificatórias da psiquiatria contemporânea.

\section{Referências}

1. Banzato CEM, Pereira ME, Santos Jr A, Silva LF, Loureiro Jr JC, Barros BR. O que os psiquiatras brasileiros esperam das classificações diagnósticas? J Bras Psiquiatr. 2007;56(2):88-93.

2. Kendell R, Jablensky A. Distinguishing between the validity and utility of psychiatric diagnoses. Am J Psychiatry. 2003;160:4-12.

3. First MB, Pincus IS, Levine JB, Williams JB, Ustun DS, Peele R. Clinical utility as a criterion for revising psychiatric diagnoses. Am J Psychiatry. 2004;161(6):946-54.

4. Jampala VC, Sierles FS, Taylor MA. Costumers views of DSM-III: Attitudes and practices of U.S. psychiatrists and psychiatric 1984 graduating residents. Am J Psychiatry. 1986;143(2):148-53.

5. Maser JD, Kaelber C, Weize DR. International use and attitudes toward DSM-III and DSM-III-R: Growing consensus in psychiatric classification. J Abnorm Psychol. 1991;100(3):271-9.

6. Bassett AS, Beiser M. DSM-III: Use of the multiaxial diagnostic system in clinical practice. Can J Psychiatry. 1991;36(4):270-4.

7. Mezzich JE. International survey on the use of ICD-10 diagnostic and related systems. Psychopathology.2002;35(2-3):72-5.

8. Banzato CE. Multiaxial diagnosis in psychiatry: Review of the literature on DSM and ICD multiaxial schemas. J Bras Psiquiatr. 2004;53(1):27-37.

9. Williams JB, Spitzer RL, Skodol AE. DSM-III in residency training: results of the National Survey. Am J Psychiatry. 1985;142(6):755-8.

10. Velamoor VR, Waring EM, Fisman S, Cernovsky Z, Brownstone D. DSM-III in residency training: results of a Canadian survey. Can J Psychiatry. 1989;34(2):103-6.

11. Mellsop G, Banzato CE, Shinfuku N, Nagamine M, Pereira ME, Dutu G. An international study of the views of psychiatrists on present and preferred characteristics of classifications of psychiatric disorders. Int J Ment Health. 2008;36(4):17-25. 